\title{
Electromagnetic properties of vibrational bands in ${ }^{170} \mathrm{Er}$
}

\author{
D.D. DiJulio ${ }^{1, a}$, J. Cederkall ${ }^{1}$, C. Fahlander ${ }^{1}$, A. Ekström ${ }^{1}$, P. Golubev ${ }^{1}$, K. Mattsson ${ }^{1}$, D. Rudolph ${ }^{1}$, G. de Angelis ${ }^{2}$, \\ S. Aydin ${ }^{3}$, A. Y. Deo ${ }^{4}$, E. Farnea ${ }^{3}$, G. Farrelly ${ }^{4}$, K. Geibel ${ }^{5}$, C. He $^{2}$, J. Iwanicki ${ }^{6}$, R. Kempley ${ }^{4}$, N. Marginean $^{7}$, \\ R. Menegazzo ${ }^{3}$, D. Mengoni ${ }^{3}$, R. Orlandi ${ }^{8}$, Z. Podolyak ${ }^{4}$, F. Recchia ${ }^{2}$, P. Reiter ${ }^{5}$, E. Sahin ${ }^{2}$, J. Smith $^{8}$, \\ P.A. Söderström ${ }^{9}$, D.A. Torres ${ }^{8}$, G.M. Tveten ${ }^{10}$, C.A. Ur ${ }^{3}$, J.J. Valiente-Dobón ${ }^{2}$, A. Wendt ${ }^{5}$, and M. Zielińska ${ }^{6}$ \\ 1 Department of Physics, Lund University, Sweden \\ 2 INFN, Laboratori Nazionali di Legnaro, Legnaro, Italy \\ 3 INFN and Università di Padova, Padova, Italy \\ 4 Department of Physics, University of Surrey, Guildford GU2 7XH, UK \\ ${ }^{5}$ University of Cologne, Cologne, Germany \\ 6 Heavy Ion Laboratory, University of Warsaw, Warsaw, Poland \\ 7 National Institute for Physics and Nuclear Engineering, Bucharest, Romania \\ 8 University of West Scotland, Paisley, Scotland, UK \\ 9 Department of Physics and Astronomy, Uppsala University, Sweden \\ 10 University of Oslo, Norway
}

Received: 4 October 2010 / Revised: 3 December 2010

Published online: 16 February 2011

(c) The Author(s) 2011. This article is published with open access at Springerlink.com Communicated by J. Äystö

\begin{abstract}
Excited states of the nucleus ${ }^{170}$ Er have been studied by Coulomb excitation using the GASP $\gamma$-ray detector system at the Laboratori Nazionali di Legnaro. The ground-state band along with a low-lying $K^{\pi}=0^{+}$band and $\gamma$-vibrational band were populated during the experiment. Based on the measured $\gamma$-ray yields, a set of interband and intraband matrix elements has been extracted using the Coulomb excitation code GOSIA. The resulting E2 matrix elements are compared to collective model predictions.
\end{abstract}

\section{Introduction}

In the nucleus ${ }^{170} \mathrm{Er}$, a low-lying $K^{\pi}=0^{+}$band occurs at nearly the same energy as the $\gamma$-vibrational band. The interaction between these bands has been investigated previously in inelastic scattering experiments [1]. While the reduced quadrupole transition probability between the ground-state band and the $2^{+}$state of the $K^{\pi}=0^{+}$band corresponds to only $\sim 8 \%$ of the transition probability to the $2^{+}$state in the $\gamma$-band [2], mixing between the two bands at spin $4^{+}$enhances the population of the low-lying $K^{\pi}=0^{+}$band. This mixing manifests itself as a deviation in the electromagnetic transition strengths from the leading-order relations described by Bohr and Mottelson [3]. Therefore a direct measure of these strengths provides information on not only the collective and single-particle properties but also on the magnitude of the mixing. For this reason we have carried out a Coulomb excitation measurement of the nucleus ${ }^{170}$ Er. Prior to this experiment, experimental evidence regarding electromagnetic transition strengths was limited to the low-lying band heads and the intraband elements

\footnotetext{
a e-mail: douglas.dijulio@nuclear.lu.se
}

of the ground-state band. A number of low-lying positiveand negative-parity bands have also been established [4, 5]. In addition, candidate states built on the two phonon $\gamma$-vibrational mode have been identified in the neighboring nuclei ${ }^{166} \operatorname{Er}[6,7]$ and ${ }^{168} \operatorname{Er}[8-10]$. Due to the low-lying nature of these bands, Coulomb excitation provides an excellent means for further exploring the electromagnetic properties and interactions of these structures.

\section{Experimental methods}

The Coulomb excitation measurement was performed using a ${ }^{32} \mathrm{~S}$ beam at a safe energy of $117 \mathrm{MeV}$ at the Laboratori Nazionali di Legnaro, Italy. The beam current throughout the experiment was $\sim 1$ pnA. The GASP [11] $\gamma$-ray detector system was used to detect the de-excitation $\gamma$-rays. GASP consists of 40 hyper-pure high-efficiency Compton-suppressed germanium detectors. The detector system was set up in configuration II, without the BGO multiplicity filter. In this configuration the array has a total efficiency of $\sim 5.8 \%$ at $1.33 \mathrm{MeV}$. A square Double-Sided Silicon Strip Detector (DSSSD), placed 
at $90^{\circ}$ with respect to the beam axis and perpendicular to the $1 \mathrm{mg} / \mathrm{cm}^{2}{ }^{170} \mathrm{Er}$ target, was used for particle identification. During the experiment, the strips of the $32 \times 32$ DSSSD array were connected in groups of two amounting to a total of 32 channels. The recording of an event was triggered by the detection of at least a single $\gamma$-ray in GASP and a charged particle in the DSSSD. The EUCLIDES [12] data acquisition system along with Mesytec [13] amplifiers were used for readout of the detectors. In addition a $12 \mu \mathrm{m}$ thick $\mathrm{Al}$ foil was placed cylindrically around the target position to stop delta electrons. A thin ${ }^{181} \mathrm{Ta}$ collimator was also placed upstream of the target. Energy calibration of the GASP array was performed using a standard ${ }^{152} \mathrm{Eu} \gamma$-ray source.

The results presented in this paper were obtained through the following steps: 1) reducing the data to events which contained only a single front and back strip hit in the DSSSD; 2) applying a time cut between front and back strip hits; 3) imposing a kinematical cut on the measured particle energies and scattering angles; 4) applying a particle- $\gamma$-ray coincidence condition; and 5) event-byevent Doppler correction of the resulting $\gamma$-ray spectrum. This last condition is necessary since the $\gamma$-rays of interest were emitted in-flight. With this selection on the kinematics of the reaction, the target thickness, and the insertion of the $\mathrm{Al}$ foil, the ${ }^{170} \mathrm{Er}$ particles are stopped before reaching the DSSSD. The energies and angles of the recoiling nucleus were calculated based on the information provided by the ${ }^{32} \mathrm{~S}$ scattered beam particles. The reconstructed angles and energies were subsequently used during the Doppler correction. A selection of the measured raw and Doppler-corrected spectra are shown in fig. 1. The data represent $\gamma$-rays detected in the full GASP array in coincidence with a particle in the region corresponding to $52.4^{\circ}<\theta<66.0^{\circ}$ of the DSSSD. In total, $\sim 3.3 \times 10^{6}$ particle $-\gamma$-ray coincidences were used in the analysis. Emitted $\gamma$-rays resulting from excitations in the ${ }^{181}$ Ta collimator and peaks from ${ }^{166-168}$ Er contaminants can also be identified in the Doppler-corrected spectrum.

A partial level scheme resulting from the experiment is shown in fig. 2. The low-lying $K^{\pi}=0^{+}$band and $\gamma$ vibrational band were excited up to the $6^{+}$states and the ground band up to the $10^{+}$state. In addition several previously known higher-lying states were excited. These include two $3^{-}$states at $1340 \mathrm{keV}$ and $1579 \mathrm{keV}$, one $5^{-}$ state at $1483 \mathrm{keV}$, one $7^{-}$state at $1705 \mathrm{keV}$, and a $2^{+}$ state at $1416 \mathrm{keV}[2]$. The placement of these states were confirmed by the coincidence data. In addition, a $686 \mathrm{keV}$ $\gamma$-ray was found in coincidence with the $843 \mathrm{keV} \gamma$-ray depopulating the $4_{K=0^{+}}^{+}$state, corresponding to a level at an energy of $1789 \mathrm{keV}$. The coincidence data showed no indication of a significant population of the $K^{\pi}=3^{+}$ band observed in ref. [1]. The width of the $1049 \mathrm{keV}$ peak was, however, measured to be $\sim 10 \%$ larger than the peak at $1024 \mathrm{keV}$, which could possibly indicate the presence of a doublet arising from the $4_{K=3^{+}}^{+} \rightarrow 4_{g}^{+}$transition.

From the Doppler-corrected $\gamma$-ray singles spectrum, relative intensities were extracted using the RADWARE $[14,15]$ package. They are listed in table 1 . Due to
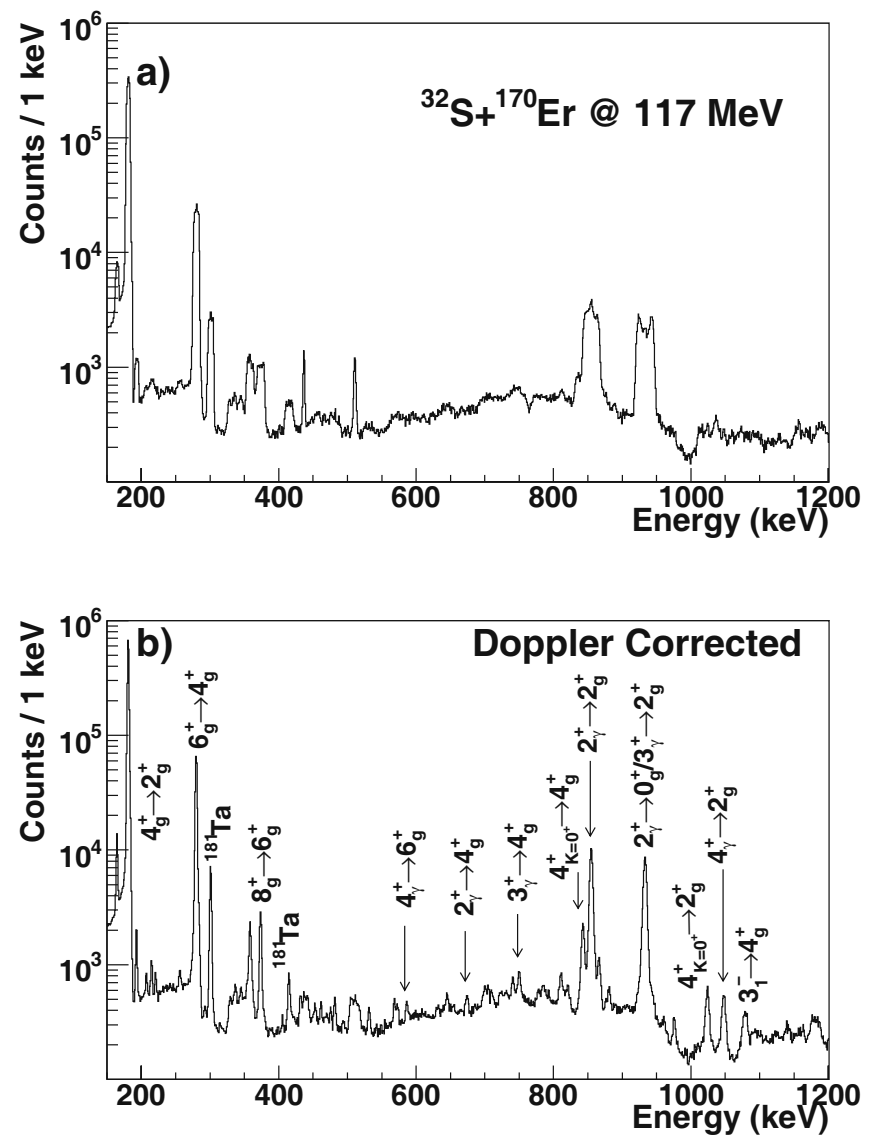

Fig. 1. Raw (a) and Doppler-corrected (b) $\gamma$-ray spectra for the entire experiment detected with the full GASP array. The spectra were generated with the coincidence condition that a scattered ${ }^{32} \mathrm{~S}$ nucleus was detected within the range $52.4^{\circ}<$ $\theta<66.0^{\circ}$. Several of the relevant transitions are highlighted. The ${ }^{181}$ Ta lines arise from the collimator placed in front of the target position.

uncertainties in the efficiency calibration at low energies, the intensities for the $4_{g}^{+} \rightarrow 2_{g}^{+}$and $2_{g}^{+} \rightarrow 0_{g}^{+}$transitions were not determined with high precision and were therefore not used directly in the analysis described below. The transitions corresponding to $2_{\gamma}^{+} \rightarrow 0_{g}^{+}$and $3_{\gamma}^{+} \rightarrow 2_{g}^{+}$, as well as $0_{K=0^{+}}^{+} \rightarrow 2_{g}^{+}$and $6_{K=0^{+}}^{+} \rightarrow 6_{g}^{+}$are given as unresolved doublets. The $4_{K=0^{+}}^{+} \rightarrow 6_{g}^{+}$transition may or may not be present in the spectrum and is given as an upper limit. The two transitions at $476 \mathrm{keV}$ and $481 \mathrm{keV}$ possibly overlap with corresponding transitions in ${ }^{181} \mathrm{Ta}$ and should be considered upper limits [16].

\section{Coulomb excitation analysis}

The yields corresponding to the relative intensities shown in table 1 can be used as input for the coupled-channels code GOSIA [17] to extract electromagnetic properties of the low-lying structures shown in fig. 2. The principle behind the code is to find a set of electromagnetic matrix elements, within the Coulomb excitation framework, which 


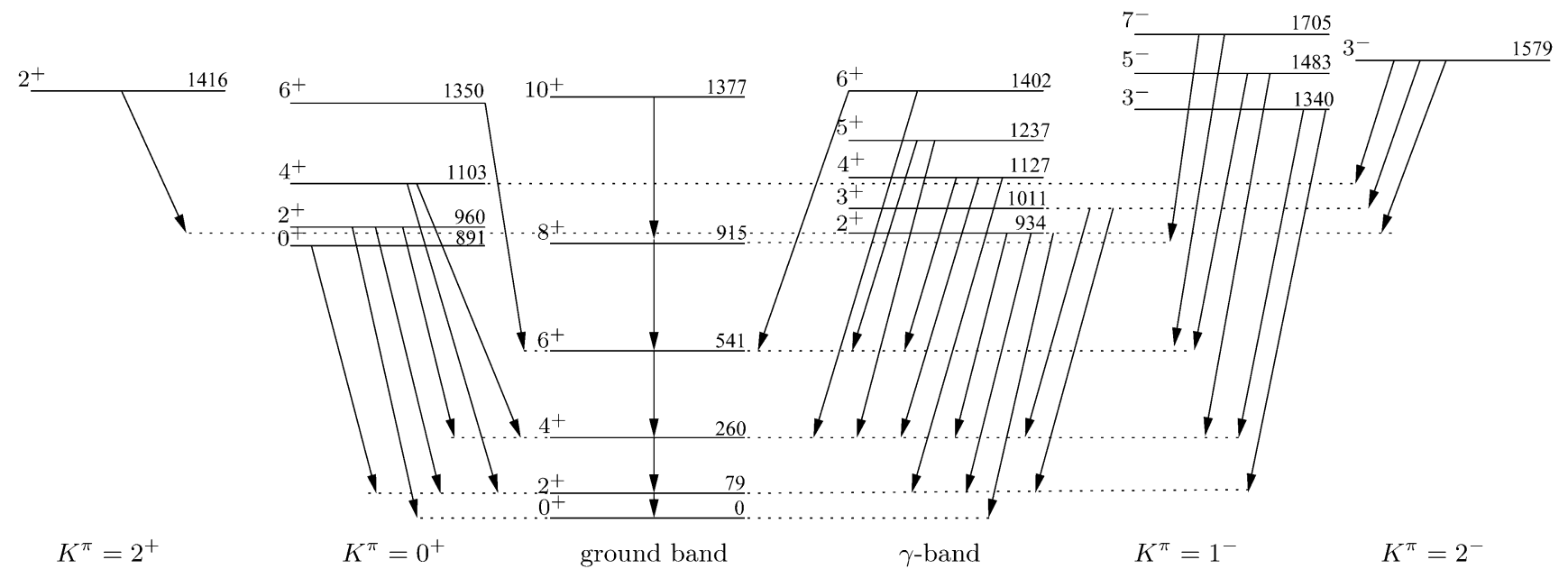

Fig. 2. Partial level scheme showing the levels observed during this study. The $K^{\pi}=0^{+}$ground-state band (central) and low-lying $K^{\pi}=0^{+}$(to the left), the $K^{\pi}=2^{+} \gamma$-band (to the right), a $K^{\pi}=2^{+}$state (far left), and negative-parity states (far right) are indicated. The transitions observed in the experiment are indicated by arrows. The level energies are given in keV.

Table 1. Relative intensities extracted from the Dopplercorrected spectrum.

\begin{tabular}{|c|c|c|}
\hline Transition & $\begin{array}{l}\text { Energy } \\
(\mathrm{keV})\end{array}$ & $\begin{array}{l}\text { Relative } \gamma \text {-ray } \\
\text { intensity }\end{array}$ \\
\hline $6_{g}^{+} \rightarrow 4_{g}^{+}$ & 280 & $100(6)$ \\
\hline $8_{g}^{+} \rightarrow 6_{g}^{+}$ & 374 & $4.88(30)$ \\
\hline $10_{g}^{+} \rightarrow 8_{g}^{+}$ & 462 & $0.37(4)$ \\
\hline $2_{\gamma}^{+} \rightarrow 0_{g}^{+} / 3_{\gamma}^{+} \rightarrow 2_{g}^{+}$ & $934 / 932$ & $42(3)$ \\
\hline $2_{\gamma}^{+} \rightarrow 2_{g}^{+}$ & 855 & $43(3)$ \\
\hline $2_{\gamma}^{+} \rightarrow 4_{g}^{+}$ & 674 & $0.55(6)$ \\
\hline $3_{\gamma}^{+} \rightarrow 4_{g}^{+}$ & 750 & $1.45(11)$ \\
\hline $4_{\gamma}^{+} \rightarrow 2_{g}^{+}$ & 1049 & $2.37(18)$ \\
\hline $4_{\gamma}^{+} \rightarrow 4_{g}^{+}$ & 867 & $3.26(22)$ \\
\hline $4_{\gamma}^{+} \rightarrow 6_{g}^{+}$ & 587 & $0.44(5)$ \\
\hline $5_{\gamma}^{+} \rightarrow 4_{g}^{+}$ & 976 & $0.72(7)$ \\
\hline $5_{\gamma}^{+} \rightarrow 6_{g}^{+}$ & 696 & $0.17(11)$ \\
\hline $6_{\gamma}^{+} \rightarrow 4_{g}^{+}$ & 1142 & $<0.62$ \\
\hline $6_{\gamma}^{+} \rightarrow 6_{g}^{+}$ & 861 & $<2.50$ \\
\hline $0_{K=0^{+}}^{+} \rightarrow 2_{g}^{+} / 6_{K=0^{+}}^{+} \rightarrow 6_{g}^{+}$ & $812 / 810$ & $1.67(16)$ \\
\hline $2_{K=0^{+}}^{+-0} \rightarrow 0_{g}^{+}$ & 960 & $0.35(7)$ \\
\hline $2_{K=0+}^{+} \rightarrow 2_{g}^{+}$ & 881 & $0.92(10)$ \\
\hline $2_{K=0^{+}}^{+} \rightarrow 4_{g}^{+}$ & 700 & $0.73(13)$ \\
\hline $4_{K=0^{+}}^{+} \rightarrow 2_{g}^{+}$ & 1024 & $2.37(17)$ \\
\hline $4_{K=0^{+}}^{+} \rightarrow 4_{g}^{+}$ & 843 & $7.96(49)$ \\
\hline $4_{K=0^{+}}^{+} \rightarrow 6_{g}^{+}$ & 563 & $<0.11$ \\
\hline $3_{1}^{-} \rightarrow 4_{g}^{+}$ & 1080 & $1.36(11)$ \\
\hline $3_{1}^{-} \rightarrow 2_{g}^{+}$ & 1262 & $<0.40$ \\
\hline $3_{2}^{-} \rightarrow 2_{\gamma}^{+}$ & 645 & $0.56(6)$ \\
\hline $3_{2}^{-} \rightarrow 3_{\gamma}^{+}$ & 569 & $0.50(6)$ \\
\hline $3_{2}^{-} \rightarrow 4_{K=0^{+}}^{+}$ & 476 & $0.26(4)^{a}$ \\
\hline $2_{K=2^{+}}^{+} \rightarrow 2_{\gamma}^{+}$ & 481 & $0.48(5)^{a}$ \\
\hline
\end{tabular}

$\bar{a}$ These lines may be contaminated with corresponding lines in ${ }^{181} \mathrm{Ta}$ and should be considered as upper limits. best reproduces the experimentally measured yields. A least-squares approach is employed and the quality of the fit is determined by the normalized $\chi^{2}$ criterion. The $\chi^{2}$ parameter is calculated in the standard way, but is however normalized to the number of data points rather than the number of degrees of freedom. The input to GOSIA, in addition to the measured yields, are the energies of the observed excited states, along with some unobserved states to account for virtual excitation effects, the geometry of the particle and $\gamma$-ray detector setup, and other previously known experimental data. These include branching ratios, lifetimes, mixing ratios, and matrix elements.

The leading-order intensity relation, described by Bohr and Mottelson [3], relates the interband and intraband matrix elements between the states shown in fig. 2 to one free parameter respectively, the intrinsic matrix element. This relation can be represented by eq. (4-92) in ref. [3],

$$
\begin{aligned}
& \left\langle K_{2} I_{2}\|\mathcal{M}(\lambda)\| K_{1} I_{1}\right\rangle= \\
& \left(2 I_{1}+1\right)^{1 / 2}\left\langle I_{1} K_{1} \lambda, \Delta K \mid I_{2} K_{2}\right\rangle\left\langle K_{2}|\mathcal{M}(\lambda, \mu)| K_{1}\right\rangle \xi,
\end{aligned}
$$

where $\left\langle K_{2}|\mathcal{M}(\lambda, \mu)| K_{1}\right\rangle$ is the intrinsic matrix element, $\left\langle K_{2} I_{2}\|\mathcal{M}(\lambda)\| K_{1} I_{1}\right\rangle$ is the reduced matrix element, and $\left\langle I_{1} K_{1} \lambda \Delta K \mid I_{2} K_{2}\right\rangle$ is the Clebsch-Gordan coefficient. For transitions between the three low-lying bands, $K_{1}=0$ and the constant $\xi=\sqrt{2}$ if $K_{2}=2$ and is equal to 1 when $K_{2}=0$. For the intraband transitions, the constant $\xi$ is also equal to 1 . Three intrinsic matrix elements, one for each of the bands, coupling the states up to spin $12^{+}$in the ground band, $10^{+}$in the $K^{\pi}=0^{+}$band, and $8^{+}$in the $\gamma$ band, were defined in the fitting procedure. Additionally, two intrinsic matrix elements for the interband transitions between the $\gamma$ - and ground bands and the $K^{\pi}=0^{+}$and ground bands were also defined. The coupling between the $\gamma$ - and $K^{\pi}=0^{+}$bands was assumed to be zero.

A similar coupling approach has also been introduced for the $M 1$ matrix elements. In principle these components could be determined from previously known mixing ratios and could potentially be entered into GOSIA. However 
Table 2. Previously known branching ratios and lifetimes defined as data points during the GOSIA fitting procedure. These branching ratios and lifetimes were reproduced in the fit within the given $1 \sigma$ limit.

\begin{tabular}{ll}
\hline$I_{i} \rightarrow I_{f} / I_{i} \rightarrow I_{f}$ & Ref. $[2]$ \\
\hline $2_{\gamma}^{+} \rightarrow 4_{g}^{+} / 2_{\gamma}^{+} \rightarrow 2_{g}^{+}$ & $0.014(3)$ \\
$2_{\gamma}^{+} \rightarrow 0_{g}^{+} / 2_{\gamma}^{+} \rightarrow 2_{g}^{+}$ & $0.897(24)$ \\
$3_{\gamma}^{+} \rightarrow 4_{g}^{+} / 3_{\gamma}^{+} \rightarrow 2_{g}^{+}$ & $0.146(11)$ \\
$4_{\gamma}^{+} \rightarrow 2_{g}^{+} / 4_{\gamma}^{+} \rightarrow 4_{g}^{+}$ & $0.86(11)$ \\
$2_{K=0^{+}}^{+} \rightarrow 4_{g}^{+} / 2_{K=0^{+}}^{+} \rightarrow 2_{g}^{+}$ & $0.65(4)$ \\
$2_{K=0^{+}}^{+} \rightarrow 0_{g}^{+} / 2_{K=0^{+}}^{+} \rightarrow 2_{g}^{+}$ & $0.63(7)$ \\
$4_{K=0^{+}}^{+} \rightarrow 2_{g}^{+} / 4_{K=0^{+}}^{+} \rightarrow 4_{g}^{+}$ & $0.273(22)$ \\
$6_{K=0^{+}}^{+} \rightarrow 4_{K=0^{+}}^{+} / 6_{K=0^{+}}^{+} \rightarrow 6_{g}^{+}$ & $0.076(25)$ \\
\hline Level & Lifetime (ps) ref. [2] \\
\hline $2_{\gamma}^{+}$ & $2.61(9)$ \\
$2_{K=0^{+}}^{+}$ & $17.46(216)$ \\
\hline
\end{tabular}

there exists a large discrepancy in both sign and magnitude for many of the mixing ratios listed in ref. [2] and they were therefore not included. The $M 1$ matrix elements were instead coupled to intrinsic matrix elements which were each varied as a single parameter during the fit. The $M 1$ intraband matrix elements for the $\gamma$-band were included by eq. (1). The $K$-forbidden $M 1$ transitions to the ground band were coupled using eq. (4-95) in ref. [3]. The $M 1$ components between the $K^{\pi}=0^{+}$and ground bands were included by eq. (4-254) in ref. [3].

This simplified coupling scheme is however not sufficient to reproduce the observed decay patterns. It is possible that the two near-degenerate $4^{+}$states in the $K^{\pi}=0^{+}$ and $\gamma$-bands lead to a breaking of the coupling scheme described above. During the fitting procedure, the interband $E 2$ matrix elements, originating from the $0^{+}, 2^{+}$and $4^{+}$ states in the $K^{\pi}=0^{+}$and $\gamma$-bands, were instead varied individually. The remaining interband $E 2$ matrix elements were coupled to the respective $2_{K=0^{+}, \gamma}^{+} \rightarrow 0_{g}^{+}$element by eq. (1) and each varied as a single parameter. This approach is similar to the procedures outlined in refs. [18, 19]. The $M 1$ and in-band E2 matrix elements were coupled as described above. The signs of the start values were selected to correspond to the signs of the Clebsch-Gordan coefficients but were allowed to vary. It should be noted that during the final error analysis, the above constraints were removed and the error calculation was performed individually for each matrix element.

The matrix elements coupling the $2^{+}$level at $1416 \mathrm{keV}$ and the four negative-parity states showed little sensitivity to the data set. Further, no reliable information regarding the level at $1416 \mathrm{keV}$ could be extracted from the Doppler-corrected spectrum. Evidence for possible band mixing effects on the $K^{\pi}=1^{-}$band were discussed in ref. [5]. The $K$-selection rules, which would normally hinder transitions from the $K^{\pi}=2^{-}$band to the $K^{\pi}=0^{+}$
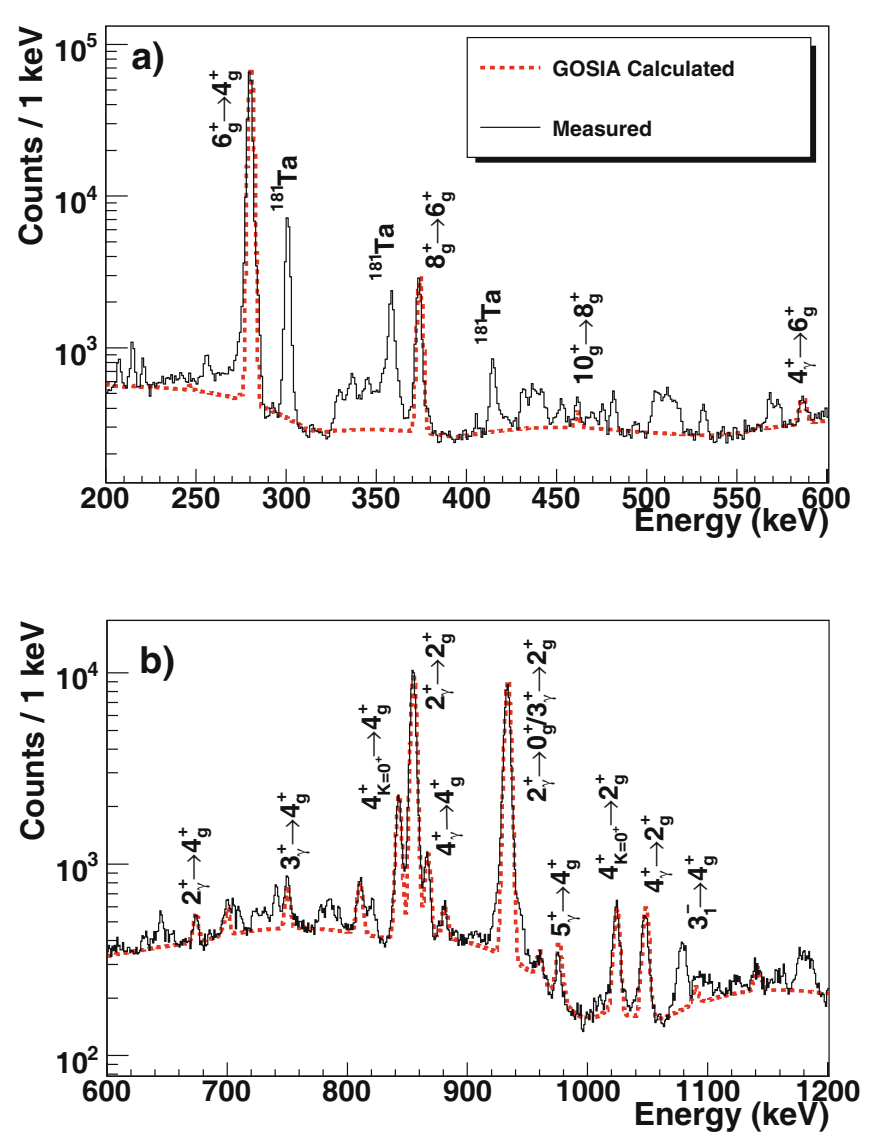

Fig. 3. Calculated spectrum based on the best set of matrix elements as determined from the GOSIA fitting procedure compared with the experimentally measured spectrum. Details related to the generation of the calculated spectrum are given in the text.

band, are broken due to the mixing of the two $4^{+}$states discussed above [5]. This conclusion is further supported by the observation of the $3_{2}^{-}$transitions shown in table 1 . These challenges make it difficult to draw firm conclusions on the properties of these structures. Therefore, the matrix elements coupling the three lowest bands were fit first and the influence of the higher-lying states was estimated as discussed below.

The coupling scheme described above reduced the number of matrix elements to 19 and a total of 31 experimental data points were defined in the fitting procedure. This includes our 21 extracted $\gamma$-ray yields between the three lowest-lying bands, eight previously reported branching ratios listed in ref. [2], and the lifetimes for the $2_{\gamma}^{+}$and $2_{K=0^{+}}^{+}$states. Lifetimes for the ground-state band were not included as constraints in the fitting procedure and instead these corresponding matrix elements are compared to the fitted results in the next section. The previously known branching ratios and lifetimes are presented in table 2 and were reproduced during the GOSIA fitting procedure to within the $1 \sigma$ limit. The best fit of the experimental data resulted in a GOSIA calculated $\chi^{2}$ value of $\sim 1.8$ and the calculated $\gamma$-ray energy spectrum is shown in fig. 3. The calculated spectrum was obtained 
Table 3. Reduced matrix elements, given in eb and $\mu_{N}$, from the GOSIA analysis determined using the coupling scheme described in the text. The error bars for each matrix element are those given by GOSIA. The IBA-1 calculations, described in the text, are given for comparison. The matrix elements marked with footnotes are compared with values from previous measurements.

\begin{tabular}{lcl}
\hline Transition & GOSIA & IBA-1 \\
\hline Inband $E 2$ transition matrix elements & \\
$2_{g}^{+} \rightarrow 0_{g}^{+}$ & $2.59_{-0.09}^{+0.05 a}$ & $2.59^{h}$ \\
$4_{g}^{+} \rightarrow 2_{g}^{+}$ & $4.15_{-0.27}^{+0.08 b}$ & 4.12 \\
$6_{g}^{+} \rightarrow 4_{g}^{+}$ & $5.23_{-0.15}^{+0.16 c}$ & 5.14 \\
$8_{g}^{+} \rightarrow 6_{g}^{+}$ & $6.12_{-0.32}^{+0.93 d}$ & 5.92 \\
$4_{\gamma}^{+} \rightarrow 2_{\gamma}^{+}$ & $2.70_{-0.08}^{+0.37}$ & 2.45 \\
$6_{\gamma}^{+} \rightarrow 4_{\gamma}^{+}$ & $4.56_{-2.52}^{+0.22}$ & 4.09 \\
$2_{K=0^{+}}^{+} \rightarrow 0_{K=0^{+}}^{+}$ & $1.91_{-0.18}^{+0.16}$ & 2.28 \\
$4_{K=0^{+}}^{+} \rightarrow 2_{K=0+}^{+}$ & $3.07_{-0.57}^{+0.40}$ & 3.62 \\
Interband $E 2$ transition $^{+}$matrix elements & \\
$2_{\gamma}^{+} \rightarrow 0_{g}^{+}$ & $0.32_{-0.01}^{+0.01 e}$ & $0.32^{h}$ \\
$3_{\gamma}^{+} \rightarrow 2_{g}^{+}$ & $-0.51_{-0.03}^{+0.11}$ & -0.50 \\
$3_{\gamma}^{+} \rightarrow 4_{g}^{+}$ & $-0.32_{-0.07}^{+0.02}$ & -0.36 \\
$6_{\gamma}^{+} \rightarrow 4_{g}^{+}$ & $0.36_{-0.27}^{+0.03}$ & 0.31 \\
$6_{\gamma}^{+} \rightarrow 6_{g}^{+}$ & $0.70_{-0.54}^{+0.53}$ & 0.70 \\
$2_{K=0^{+}}^{+} \rightarrow 0_{g}^{+}$ & $0.09_{-0.01}^{+0.01 f}$ & 0.12
\end{tabular}

Interband $M 1$ transition matrix elements

$$
2_{K=0^{+}}^{+} \rightarrow 2_{g}^{+} \quad|0.10|_{-0.01}^{+0.01 g}
$$

$\bar{a}$ Previously measured using Coulomb excitation to 2.33(3), 2.48(9), $2.41(2)$ see refs. [20-22], respectively.

${ }^{b}$ Previously measured to 3.74(5) [23].

${ }^{c}$ Previously measured to $5.00(11)$ [23].

${ }^{d}$ Previously measured to $5.66(21)[23]$ and to $\pm 5.93(24)$ ref. [2].

$e^{e}$ Reported value $\pm 0.321(5)$ in ref. [2].

$f$ Reported value $\pm 0.088(5)$ in ref. [2].

$g$ Reported value $\pm 0.099(8)$ in ref. [2].

$h$ This matrix element was used to normalize the results of the IBA-1 model calculation.

by fitting the background of the Doppler-corrected spectrum shown in fig. 1 and adding the GOSIA calculated yields. The yields were Gaussian broadened to meet the experimentally measured FWHM as a function of energy. The $4_{K=0^{+}}^{+} \rightarrow 2_{g}^{+}$transition was used to normalize the two spectra to each other.

\section{Results and discussion}

A reduced set of matrix elements with correlated errors was determined as described above. They are shown in tables 3 and 4 . The matrix elements indicated with foot-
Table 4. Individually fitted reduced matrix elements, given in eb, from the GOSIA analysis. The errors are those given from GOSIA. The predictions of eq. (1) along with the IBA-1 calculations, discussed in detail in the text, are given for comparison. The predictions of eq. (1) were calculated using the $2^{+} \rightarrow 0_{g}^{+}$matrix elements for the two bands given in table 3 . The matrix elements marked with footnotes are compared with previous measurements.

\begin{tabular}{|c|c|c|c|}
\hline Transition & GOSIA & eq. (1) & IBA-1 \\
\hline \multicolumn{4}{|c|}{ Interband $E 2$ transition matrix elements } \\
\hline $2_{\gamma}^{+} \rightarrow 2_{g}^{+}$ & $0.41_{-0.01}^{+0.01 a}$ & 0.38 & 0.40 \\
\hline $2_{\gamma}^{+} \rightarrow 4_{g}^{+}$ & $0.09_{-0.01}^{+0.01 b}$ & 0.09 & 0.10 \\
\hline $4_{\gamma}^{+} \rightarrow 2_{g}^{+}$ & $0.14_{-0.01}^{+0.01}$ & 0.33 & 0.31 \\
\hline $4_{\gamma}^{+} \rightarrow 4_{g}^{+}$ & $-0.05_{-0.07}^{+0.07}$ & 0.57 & 0.59 \\
\hline $4_{\gamma}^{+} \rightarrow 6_{g}^{+}$ & $0.25_{-0.02}^{+0.02}$ & 0.17 & 0.21 \\
\hline $0_{K=0^{+}}^{+} \rightarrow 2_{g}^{+}$ & $0.04_{-0.01}^{+0.01}$ & 0.09 & 0.13 \\
\hline $2_{K=0^{+}}^{+} \rightarrow 2_{g}^{+}$ & $-0.05_{-0.03}^{+0.03 c}$ & -0.11 & -0.15 \\
\hline $2_{K=0^{+}}^{+} \rightarrow 4_{g}^{+}$ & $0.22_{-0.02}^{+0.01 d}$ & 0.14 & 0.22 \\
\hline $4_{K=0^{+}}^{+} \rightarrow 2_{g}^{+}$ & $0.33_{-0.01}^{+0.01}$ & 0.14 & 0.17 \\
\hline $4_{K=0^{+}}^{+} \rightarrow 4_{g}^{+}$ & $-0.96_{-0.05}^{+0.04}$ & -0.14 & -0.19 \\
\hline $\begin{array}{l}\text { Reported value }> \\
\text { Reported value } \pm \\
\text { Reported value } \mid 0 \\
\text { Reported value } \pm\end{array}$ & $\begin{array}{l}13 \text { in ref. [2]. } \\
\text { (1) in ref. [2]. } \\
+0.03 \text { in ref. [2]. } \\
-0.02 \text {. } \\
9(14) \text { in ref. [2]. }\end{array}$ & & \\
\hline
\end{tabular}

notes are compared to previous measurements in order to evaluate the fit. The value of the $2_{g}^{+} \rightarrow 0_{g}^{+}$matrix element is compared with three other Coulomb excitation measurements [20-22]. The deviation between the two measurements, reported with the highest accuracy, of $\left\langle 2_{g}^{+}\|E 2\| 0_{g}^{+}\right\rangle=2.33(3)$ and $\left\langle 2_{g}^{+}\|E 2\| 0_{g}^{+}\right\rangle=2.41(2)$ is $\sim 3-4 \sigma$. Our measurement deviates from the largest previously reported value of $\left\langle 2_{g}^{+}\|E 2\| 0_{g}^{+}\right\rangle=2.48(9)$, by $\sim 1 \sigma$. The interband matrix elements are in good agreement with previously reported values.

In table 4 the predictions of eq. (1) are also shown for comparison. The largest deviations for the individually varied matrix elements from the predictions of eq. (1) are for the $4^{+}$state transitions of the $K^{\pi}=0^{+}$band and $\gamma$-band. The $4_{K=0^{+}}^{+} \rightarrow 4_{g}^{+}$transition is enhanced significantly and exhibits a matrix element corresponding to a transition probability of $B\left(E 2,4_{K=0^{+}}^{+} \rightarrow 4_{g}^{+}\right)=$ $18_{-2}^{+2}$ W.u. This is possibly due to the mixing discussed below. The E2/M1 mixing ratio for this transition, extrapolated from the $M 1$ matrix element in table 3 and eq. (4-254) in ref. [3] is $|2.67|_{-0.26}^{+0.19}$. This value is similar to the previously reported value of $+2.81(10)$ [24]. The current analysis was not sensitive to the sign of the $M 1$ matrix element.

The width of the final $\chi^{2}$ minimum was investigated by randomly selecting the initial values of the $M 1$ intrinsic matrix elements along with the uncoupled E2 interband 
matrix elements and repeating the minimization several times. The sign conventions were first kept as described above but were later allowed to vary during the minimization. The procedure revealed the existence of other local minima but each with a higher $\chi^{2}$ value than the best set presented here. The phases of the individually fitted matrix elements were evaluated by flipping the signs and restarting the minimization. In the majority of the cases, flipping the sign of a single-matrix element in the final set resulted in a larger $\chi^{2}$ value or no significant change in the $\chi^{2}$ value at the resulting minimum. The only exception being the sign of the $2_{\gamma}^{+} \rightarrow 2_{g}^{+}$matrix element which resulted in a slightly lower $\chi^{2}$ value but no significant change in the measured matrix elements. The positive sign is however preferred as this choice agrees with both eq. (1) and the IBA-1 calculations described below.

The influence of the three higher-lying bands on the $K^{\pi}=0^{+}, \gamma$ - and ground-state band matrix elements was investigated by fixing the values coupling these transitions to the lower bands and observing changes in the final set of matrix elements. All states indicated in fig. 2 were included in the procedure along with an additional $5^{-}$state in the $K^{\pi}=2^{-}$band. Estimates have been used to set the values of these matrix elements using data from nearby nuclei when possible. Little effect on the best set of matrix elements was observed.

Calculations using the IBA-1 model have been carried out using the program packages PHINT and FBEM [25]. Previously, comparisons with measured reduced matrix elements for the $2^{+}$states in ${ }^{170} \mathrm{Er}$ and the IBA-1 model were carried out in ref. [26]. It is now possible to extend the comparison to higer-lying states in both bands. The parameters used in the calculations are the following; $\mathrm{PAIR}=1.06 \mathrm{keV}, \mathrm{ELL}=17.08 \mathrm{keV}, \mathrm{QQ}=-21.23 \mathrm{keV}$ as given in ref. [26]. The parameters E2SD and E2DD were calculated using the experimental $B(E 2)$ values for the transitions $2_{g}^{+} \rightarrow 0_{g}^{+}$and $2_{\gamma}^{+} \rightarrow 0_{g}^{+}$. Their values were: $\mathrm{E} 2 \mathrm{SD}=0.1565 \mathrm{eb}$, and E2DD $=-0.162 \mathrm{eb}$. The calculated energy levels are in excellent agreement with the energy levels for the $\gamma$ - and $K^{\pi}=0^{+}$bands shown in fig. 2, with the largest deviation between the $6_{K=0^{+}}^{+}$energies of $\sim 60 \mathrm{keV}$. The resulting IBA-1 matrix elements are listed in tables 3 and 4 . The majority of the interband matrix elements are well accounted for by the IBA-1 calculations. There however remains a discrepancy for the $4^{+}$states of the $K=0^{+}$and $\gamma$-bands.

One unique feature of the IBA-1 model, compared to the geometric collective model, is the prediction of collective transition probabilities between the lowest-lying $K^{\pi}=0^{+}$band and the $\gamma$-band. The influence of including matrix elements between these two bands has been explored further. The minimization was restarted using the final set of matrix elements and fixing an intrinsic matrix element between the $K^{\pi}=0^{+}$and $\gamma$-bands of $\left\langle K_{2}=2|E 2| K_{1}=0\right\rangle=|0.16|$. This matrix element was calculated from the $2_{\gamma}^{+} \rightarrow 0_{K=0^{+}}^{+}$predictions of the IBA-1 model. The individual interband matrix elements were assumed to follow the relation provided by eq. (1). The most significant effect on the final set of matrix elements was observed for the $0_{K=0^{+}}^{+} \rightarrow 2_{g}^{+}$matrix element, which changed by a maximum of 0.03 eb depending on the sign choice for the intrinsic matrix element connecting the two bands. No significant influence on the remaining matrix elements was observed.

The behavior of the interband matrix elements shown in table 4 can be discussed in terms of the band mixing between the three lowest-lying bands. The E2 matrix elements between the $\gamma$ - and ground-state bands are well correlated by eq. (1). The only large deviations being for the $4_{\gamma}^{+}$transitions. These deviations could be the result of the near degeneracy of the two $4_{K=0^{+}, \gamma}^{+}$states as was discussed in ref. [1]. The fact that the interband $E 2$ matrix elements of the $\gamma$-band are well accounted for by eq. (1) indicates that the mixing between the $\gamma$ - and ground-state bands is rather weak. A similar qualitative analysis for the $K^{\pi}=0^{+}$band transitions is more difficult due to the limited number of experimentally determined matrix elements.

\section{Summary}

Coulomb excitation has been used to investigate electromagnetic properties of the nucleus ${ }^{170} \mathrm{Er}$. The $K^{\pi}=0^{+}$ band and $K^{\pi}=2^{+}, \gamma$-vibrational band were strongly populated during the experiment. In addition several lowlying octupole vibrations and a $K^{\pi}=2^{+}$state were populated. A set of matrix elements coupling the three lowestlying bands was deduced using the Coulomb excitation code GOSIA.

We would like to thank Prof. Olaf Scholten for his help in using the PHINT code package and the accelerator staff at LNL for providing technical support during the experiment. This work was supported by the European Community FP6 - Structuring the ERA - Integrated Infrastructure Initiativecontract EURONS No. RII3-CT-2004-506065.

Open Access This article is distributed under the terms of the Creative Commons Attribution Noncommercial License which permits any noncommercial use, distribution, and reproduction in any medium, provided the original author(s) and source are credited.

\section{References}

1. C.Y. Wu et al., Phys. Rev. C 61, 021305(R) (2000).

2. C.M. Baglin, Nucl. Data Sheets 96, 611 (2002).

3. A. Bohr, B. Mottelson, Nuclear Structure, Vol. II (Benjamin, Reading, MA, 1975).

4. E.P. Grigoriev, Phys. Part. Nucl. 31, 764 (2000).

5. E.P. Grigoriev, I.A. Gladkova, Phys. At. Nucl. 63, 705 (2000).

6. C. Fahlander et al., Phys. Lett. B 388, 475 (1996).

7. P.E. Garrett et al., Phys. Rev. Lett. 78, 4545 (1997).

8. H.G. Börner et al., Phys. Rev. Lett. 66, 691 (1991).

9. M. Oshima et al., Phys. Rev. C 52, 3492 (1995). 
10. T. Härtlein et al., Eur. Phys. J. A 2, 253 (1998).

11. C. Rossi Alvarez, Nucl. Phys. News 3, 3 (1993).

12. A. Gadea et al., LNL Annual Report, LNL-INFN (REP)160/00, 151 (1999).

13. http://www.mesytec.com/.

14. http://radware.phy.ornl.gov/.

15. D.C. Radford, Nucl. Instrum. Methods A 361, 297 (1995).

16. S.-C. Wu, Nucl. Data Sheets 106, 367 (2005).

17. T. Czosnyka et al., Bull. Am. Phys. Soc. 28, 745 (1983).
18. C.Y. Wu et al., Nucl. Phys. A 533, 359 (1991).

19. C.Y. Wu et al., Phys. Rev. C 64, 064317 (2001).

20. B. Elbek et al., Nucl. Phys. 19, 523 (1960).

21. R. Graetzer et al., Phys. Rev. 129, 1772 (1963).

22. K.A. Erb et al., Phys. Rev. Lett. 29, 1010 (1972)

23. S.H. Sie, D.W. Gebbie, Nucl. Phys. A 289, 217 (1977)

24. A.M. Demidov et al., Phys. At. Nucl. 62, 1271 (1999).

25. O. Scholten, private communication.

26. F.K. McGowan, Phys. Rev. C 24, 1803 (1981). 